\title{
ESTIMATING FIELD QUALITY IN LOW-BETA SUPERCONDUCTING QUADRUPOLES AND ITS IMPACT ON BEAM STABILITY
}

\author{
E. Todesco, B. Bellesia, J. P. Koutchouk, CERN, Geneva, Switzerland \\ C. Santoni, University of Clermont Ferrand, France
}

\section{Abstract}

Random components of field harmonics in superconducting quadrupoles are usually estimated by computing the effect of a random positioning of the coil blocks with an r.m.s. of $0.05 \mathrm{~mm}$. Here, we review the experience acquired in the construction of 7 superconducting quadrupoles in the RHIC and in the LHC projects to estimate the reproducibility in the coil positioning. Post-processed data show that the reproducibility is around $0.020 \mathrm{~mm}$ r.m.s., and independent of the aperture. Using this result, we work out a scaling law for the random components as a function of the magnet aperture. As an application, we analyse the impact on geometrical aberrations of the expected field errors in presence of large beta functions in the triplet.

\section{INTRODUCTION}

The low- $\beta$ insertion in the interaction region of the Large Hadron Collider (LHC) consists of a triplet of superconducting quadrupoles [1] to focus the beam in the interaction point (IP). In the baseline, one has a beta function in the IP $\beta^{*}=0.55 \mathrm{~m}$; this gives in the triplet a $\beta$ function of $4400 \mathrm{~m}$, requiring a quadrupole aperture of 70 $\mathrm{mm}$. Studies about how to improve the LHC luminosity have been started since 2002 [2-11]; a possible way is to decrease $\beta^{*}$ to values of $0.25 \mathrm{~m}$ or even less, thus producing larger beta functions in the triplet and requiring larger apertures. For instance, $90 \mathrm{~mm}$ aperture $\mathrm{Nb}_{3} \mathrm{Sn}$ magnets are being built in the framework of the LHC Accelerator Research Program (LARP) [12]. Recent studies focused on lay-outs with even larger apertures [611]. The aperture can be used not only for allowing a larger $\beta$ function in the triplet, giving a smaller $\beta^{*}$, but also to have an additional shielding to prevent power deposition in the magnet coils. Moreover, it allows increasing the collimator gap, thus reducing the impedance that presently is one of the limits of the LHC performance [13]. The natural question that arises is weather, having very large beta functions, the geometric aberrations in the large aperture quadrupoles can become critical. In order to be able to evaluate this issue, a reliable estimate of the field errors is necessary.

Here, we analyze data relative to the LHC and Relativistic Heavy Ion Collider [14] (RHIC) magnets to find the dependence of field errors on the magnet aperture, following the approaches outlined in [15-19]. We then estimate how the obtained scaling for the errors affects geometric aberrations in collision optics, where the contribution of the low- $\beta$ quadrupoles is dominant. An 07 Accelerator Technology Main Systems extended version of the paper can be found in [20]

\section{FIELD QUALITY IN RHIC AND LHC QUADRUPOLES}

We analyze the data relative to 7 types of $\mathrm{Nb}-\mathrm{Ti}$ quadrupoles that have been built for the RHIC [14] and for the LHC projects. Apertures (defined as bore diameter), operational gradients, peak fields, and temperatures are listed in Table 1.

Table 1: Features of superconducting quadrupoles in LHC and RHIC

\begin{tabular}{ccccccc}
\hline \hline & Number & $\begin{array}{c}\text { Aperture } \\
(\mathrm{mm})\end{array}$ & Layers & $\begin{array}{c}\text { G op. } \\
(\mathrm{T} / \mathrm{m})\end{array}$ & $\begin{array}{c}\text { Peak field op. } \\
(\mathrm{T})\end{array}$ & $\begin{array}{c}\mathrm{T} \\
(\mathrm{K})\end{array}$ \\
\hline RHIC MQ & 420 & 80 & 1 & 71 & 3.4 & 4.2 \\
RHIC Q1-3 & 72 & 130 & 1 & 48 & 3.8 & 4.2 \\
LHC MQ & 392 & 56 & 2 & 223 & 6.8 & 1.9 \\
LHC MQM & 86 & 56 & 2 & $200 / 160$ & $6.3 / 5.0$ & $1.9 / 4.2$ \\
LHC MQY & 24 & 70 & 4 & 160 & 6.1 & 4.2 \\
LHC MQXA & 16 & 70 & 4 & 215 & 8.4 & 1.9 \\
LHC MQXB & 16 & 70 & 2 & 215 & 8.2 & 1.9 \\
\hline \hline
\end{tabular}

The magnetic field in a quadrupole is expressed as a multipolar expansion

$$
B_{y}+i B_{x}=10^{-4} B_{2} \sum_{n=2}^{\infty}\left(b_{n}+i a_{n}\right)\left(\frac{x+i y}{R_{r e f}}\right)^{n+1}
$$

where $b_{2}=10^{4}, B_{2}$ is the main component in $\mathrm{T}$, and $R_{\mathrm{ref}}$ is a reference radius, usually set as one third of the aperture diameter, to have dimensionless multipoles $\left(b_{n}, a_{n}\right)$.

For large productions (RHIC MQ and LHC MQ) we checked that the spread of the room temperature measurements, carried out on all the magnets, and the 1.9/4.2 K measurements in collision, carried out on a limited sample (25\% for RHIC, $10 \%$ for LHC) was similar. This confirms that the random component of the multipoles is mainly due to the reproducibility of the cable positioning in the magnet aperture. Please note that this reproducibility is not related to the absolute precision in positioning the coil with respect to the design: we always assume that after a few iterations in the coil design, one manages to obtain the target values for the field harmonics. Under this assumption, the random component is the ultimate limit to a perfect field quality.

Using the approach outlined in $[17,18]$, we postprocessed the spread of the measured multipoles to evaluate the standard deviation of the reproducibility in the positioning of the coil blocks. For each coil lay-out we carry out a simulation where each coil block is randomly moved with an r.m.s. amplitude of $d_{\mathrm{s}}=0.1 \mathrm{~mm}$, and the corresponding spread in the multipoles $\sigma_{\mathrm{bn}}^{\mathrm{s}}, \sigma_{\mathrm{an}}^{\mathrm{s}}$ are

T10 Superconducting Magnets 
calculated. The multipole spread is linear in $d_{\mathrm{s}}$ over the range of interest $(0.01$ to $0.10 \mathrm{~mm})$. We define the discrepancy between the measured values $\left(\sigma_{\text {bn }}^{\mathrm{m}}, \sigma_{\text {an }}^{\mathrm{m}}\right)$ and the simulated values with an r.m.s. amplitude $d$ :

$\chi^{2}(d)=\frac{1}{2(N-2)} \sum_{n=3}^{N}\left[\log \left(\frac{d}{\sigma_{b n}^{m}} \frac{\sigma_{b n}^{s}}{d_{s}}\right)+\log \left(\frac{d}{\sigma_{a n}^{m}} \frac{\sigma_{a n}^{s}}{d_{s}}\right)\right]^{2}$

Minimizing the discrepancy with respect to $d$, we obtain the standard deviation $d_{0}$ of the reproducibility of coil positioning that best fits the measured multipole spread

$$
d_{0}=d_{s} \exp \left\{\frac{1}{2(N-2)} \sum_{n=3}^{N}\left[\log \left(\frac{\sigma_{b n}^{m}}{\sigma_{b n}^{s}}\right)+\log \left(\frac{\sigma_{a n}^{m}}{\sigma_{a n}^{s}}\right)\right]\right\}
$$

and we define the error of the fit as

$$
\varepsilon=\frac{1}{N-2} \sum_{n=3}^{N}\left\{\left|1-\frac{d_{0} \sigma_{b n}^{s}}{d_{s} \sigma_{b n}^{m}}\right|+\left|1-\frac{d_{0} \sigma_{a n}^{s}}{d_{s} \sigma_{a n}^{m}}\right|\right\} .
$$

LHC and RHIC data have been analyzed using this approach, and the reproducibility $d_{0}$ have been worked out (see Figs. 1 and 2), with $\mathrm{N}=8$.

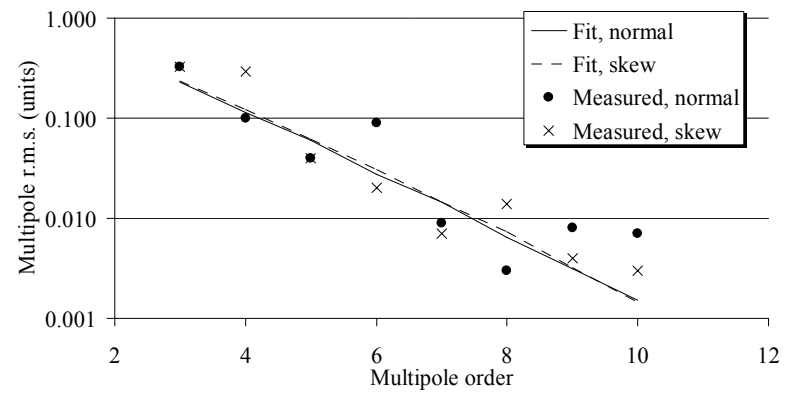

Figure 1: Measured r.m.s. of multipole in LHC MQXA at $1.9 \mathrm{~K}$ (markers), and best fit through simulations with $d_{0}=0.011 \mathrm{~mm}$

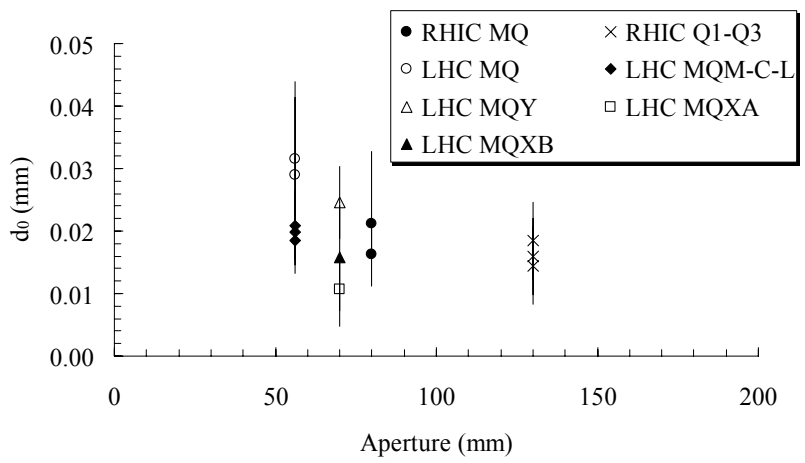

Figure 2: Reproducibility $d_{0}$ of coil positioning versus aperture derived from magnetic measurements of 7 types of superconducting quadrupoles.

One finds out that $d_{0}$ ranges from 0.010 to $0.030 \mathrm{~mm}$, without an clear dependence on the aperture: quadrupoles with a $56 \mathrm{~mm}$ aperture have a reproducibility of 0.020 $\mathrm{mm}$ (LHC MQM types) to $0.030 \mathrm{~mm}$ (LHC MQ types).
$70 \mathrm{~mm}$ aperture quadrupoles have $d_{0} \sim 0.010$ to $0.025 \mathrm{~mm}$, RHIC $80 \mathrm{~mm}$ and $130 \mathrm{~mm}$ apertures have $d_{0} \sim 0.015 \mathrm{~mm}$.

\section{A SCALING LAW FOR FIELD ERRORS}

Let us consider a quadrupole of aperture $\phi$, coil thickness $w$, and characterized by a set of random field components $\sigma$ generated by a reproducibility of coil position with r.m.s. $d_{0}$. We set the reference radius $R_{\text {ref }}$ as $1 / 3$ of the aperture diameter. The multipoles defined in (1) are invariant under a rescaling of the coordinates and of the reference radius. Therefore, if we increase the aperture by a factor $\alpha$, and consequently multiply the coil thickness, the reference radius, and the position reproducibility by the same factor, the random field components are invariant:

$$
\sigma\left(b_{n}, a_{n} ; \alpha \phi, \alpha d, \alpha R_{r e f}\right)=\sigma\left(b_{n}, a_{n} ; \phi, d, R_{r e f}\right) .
$$

The analysis of the data carried out in the previous section suggests that the reproducibility in coil positioning is independent of the coil aperture; therefore one has

$$
\sigma\left(b_{n}, a_{n} ; \alpha \phi, d, \alpha R_{r e f}\right)=\frac{1}{\alpha} \sigma\left(b_{n}, a_{n} ; \phi, d, R_{r e f}\right)
$$

i.e., the multipole spread scales with the inverse of the aperture radius. Please note that if we keep the same reference radius we have

$$
\sigma\left(b_{n}, a_{n} ; \alpha \phi, d, R_{r e f}\right)=\frac{1}{\alpha^{n}} \sigma\left(b_{n}, a_{n} ; \phi, d, R_{r e f}\right)
$$

This simple scaling is strictly valid for quadrupoles of identical cross-sections. In practice, increasing the quadrupole aperture has a significant impact on the crosssection. We verified that this impact does not change the scaling (6-7) on four realistic quadrupole cross-sections, with optimized field quality, and apertures ranging from $50 \mathrm{~mm}$ to $200 \mathrm{~mm}$. More details can be found in [20].

\section{IMPACT ON BEAM DYNAMICS}

We showed that large aperture quadrupoles are expected to have a better field quality; in this section we study the effect of this improvement on the beam dynamics in the collision optics, where, due to the high value of the $\beta$-functions, the contribution of low- $\beta$ quadrupoles is dominant.

We first consider the $1^{\text {st }}$ order amplitude-dependent tuneshift induced by the octupolar term $b_{4}$ of an IR quadrupole, which is proportional to

$$
\Delta Q \propto \int K_{3} \beta^{2} d s,
$$

where $K_{3}$ is given by

$$
K_{3} \equiv \frac{1}{B \rho} \frac{\partial^{3} B_{y}}{\partial x^{3}}=3 ! \frac{B_{2}}{B \rho} \frac{b_{4}}{R_{r e f}^{3}}=3 ! \frac{G}{B \rho} \frac{b_{4}}{R_{\text {ref }}^{2}}
$$

and $G=B_{2} / R_{\text {ref }}$ is the nominal field gradient in $\mathrm{T} / \mathrm{m}$. Therefore one has

$$
\Delta Q \propto \int K_{3} \beta^{2} d s \propto \beta_{\max }^{2} \frac{\bar{b}_{4}}{R_{r e f}^{2}} \int G d s
$$


where we approximated the integral, extracting the average values of the multipole and of the maximum of the beta function.

For a term of order $n$ one has

$$
T_{n} \propto \beta_{\max }^{\frac{n+1}{2}} \frac{\bar{b}_{n+1}}{R_{\text {ref }}{ }^{n-1}} \int G d s .
$$

If we increase the aperture by a factor $\alpha$

$$
\phi \rightarrow \alpha \phi
$$

and the reference radius

$$
R_{\text {ref }} \rightarrow \alpha R_{\text {ref }},
$$

according to the results of the previous section, the multipoles rescale according to

$$
b_{4} \rightarrow \frac{b_{4}}{\alpha}
$$

We assume as a first order approximation that the integrated gradient is constant. This is true only if the length of the quadrupole does not change. In general, a larger aperture requires a longer triplet and this reduces the integrated gradient $[10,11]$.

We now assume that the beta function is increased by a factor $\delta$

$$
\beta \rightarrow \delta \beta
$$

The nonlinear term scales as

$$
T_{n} \rightarrow \delta^{\frac{n+1}{2}} \alpha^{-n} T_{n}
$$

We can distinguish three cases.

- If the aperture is increased without increasing the beta function $(\delta=1)$, the nonlinear terms decrease with $\alpha^{n}$ and obviously the dynamics gets more stable.

- Since the beam size is proportional to the square root of the beta function, one could think about increasing the aperture as the square of the beta function $\alpha^{2}=\delta$. According to (16), in this case the aberrations grow linearly with $\alpha$, and can become unmanageable.

- Equation (16) suggests that if one considers the same increase in the beta function and in the aperture $\alpha=\delta$, one still gets decreasing nonlinear terms for larger apertures.

As an application of this scaling to the LHC upgrade, we showed in [11] that an aperture increase of $\alpha=1.9$, i.e. going from 70 to $130 \mathrm{~mm}$, with a beta function increase of $\delta=2.8$ (from $4400 \mathrm{~m}$ to $12600 \mathrm{~m}$ ), provides an increase of nonlinear terms of $30 \%$ only.

\section{CONCLUSIONS}

In this work we gave scaling laws for evaluating the expected field quality in superconducting quadrupoles. We analyzed magnetic measurements relative to the production of the LHC and RHIC quadrupoles, showing that data support the hypothesis that the reproducibility in positioning the coil, which is the main source of the random components, is independent of the magnet aperture size. Using this result, we proved that if the reference radius is fixed at $1 / 3$ of the coil aperture, the multipoles are inversely proportional to the aperture size (see Eq. 6).

We then used these scaling laws to derive the impact of large aperture low- $\beta$ insertions on geometric aberrations: if all the aperture is used to house a larger beam, geometric aberrations grow at least proportionally to the aperture. On the other hand, if the larger aperture is used to have additional shielding, the aberrations will rapidly decrease with a power of the inverse of the aperture size. We showed that increasing the beta functions and the apertures of the similar factors the geometric aberrations are not increased.

We acknowledge the support of the European Community-Research Infrastructure Activity under the FP6 "Structuring the European Research Area" program (CARE, contract number RII3-CT-2003-506395)

\section{REFERENCES}

[1] AA. VV. "LHC Design Report", CERN 2004-003 (2004)

[2] O. Bruning, et.al., "LHC Luminosity and energy upgrade: a feasibility study", LHC Project Report 626 (2002).

[3] T. Sen, et al., "Beam physics issues for a possible 2nd generation LHC IR“, European Particle Accelerator Conference (2002) 3713 .

[4] J. Strait, et al., "Towards a new LHC interaction region design for luminosity upgrad“, Particle Accelerator Conference (2003) 42-5.

[5] F. Ruggiero, et al., " Performance Limits and IR Design of a Possible LHC Luminosity Upgrade Based on Nb-Ti SC Magnet Technology“ European Particle Accelerator Conference (2004) 608-10.

[6] R. Ostojic, et al., "Low- $\beta$ quadrupole designs for the LHC luminosity upgrade“, Particle Accelerator Conference (2005) 2795-7.

[7] J.P. Koutchouk, "Investigations of the Parameter Space for the LHC Luminosity Upgrade”, LHC Project Report 973 (2006).

[8] R. De Maria, O. Bruning, "A Low Gradient Triplet Quadrupole Layout Compatible with NbTi Magnet Technology and $\beta *=0.25$ cm”, European Particle Accelerator Conference (2006) 574-6.

[9] R. De Maria, CARE proceedings, Valencia (2006).

[10] E. Todesco, J.P. Koutchouk, CARE proceedings, Valencia (2006).

[11] J.P.Koutchouk, L. Rossi, E. Todesco, LHC Project Report 1000 (2007).

[12] S. Gourlay, et at, "Magnet R\&D for the US LHC Accelerator Research Program (LARP)", IEEE Trans. Appl. Supercond. 16 (2006) 324-7.

[13] R. Assman, CARE proceedings, Valencia (2006).

[14] M. Anerella et al., "The RHIC magnet system", Nucl. Instrum. Meth. A499 (2003) 280-315.

[15] J. Herrera et al., "Random errors in the magnetic field coefficients of superconducting magnets",.Particle Accelerator Conference (1985) 3689-91.

[16] R. Gupta, "Estimating and adjusting field quality in superconducting magnets", Part. Accel. 55 (1996) 129-39.

[17] W. Scandale, E. Todesco, R. Wolf, "Random errors induced in the superconducting windings in the LHC dipoles", IEEE Trans. Appl. Supercond. 10 (2000) 93-7.

[18] P. Ferracin, W. Scandale, E. Todesco, R. Wolf, "Modelling of random geometric errors in superconducting magnets with applications to the Large Hadron Collider", Phys. Rev. ST-AB 3 (2000) 122403.

[19] B. Bellesia, C. Santoni, E. Todesco, "Random errors in superconducting dipoles", European Particle Accelerator Conference (2006).

[20] B. Bellesia, J. P. Koutchouk, E. Todesco, Phys. Rev. STAB, 10 (2007) 062401. 